Publicaciones 



\section{Publicaciones Centro de Derechos Humanos}

Las publicaciones del Centro de Derechos Humanos son de acceso universal y gratuito. Están disponibles en www.cdh.uchile.cl

\section{Publicaciones Periódicas}

Anuario de Derechos Humanos, Nos 1 a 9, años 2005-2013.

Boletín de Jurisprudencia de la Corte Interamericana de Derechos Humanos, 2009-2013.

\section{Libros}

AGUILÓ, Pedro; MILOS, Catalina, NASH, Claudio (Investigadores). Personas privadas de libertad y medidas disciplinarias en Chile: análisis y propuestas desde una perspectiva de derechos humanos. Santiago de Chile: Centro de Derechos Humanos, Facultad de Derecho, Universidad de Chile, 2013.

NASH, Claudio. Derecho Internacional de los Derechos Humanos en Chile. Recepción y aplicación en el ámbito interno. Santiago de Chile: Centro de Derechos Humanos, Facultad de Derecho, Universidad de Chile, 2012.

Centro de Derechos Humanos. Estudio Acceso a la Justicia en el Sistema Interamericano de Derechos Humanos: Transparencia y Representación Legal. Santiago de Chile: Centro de Derechos Humanos, Facultad de Derecho, Universidad de Chile, 2012.

Centro de Derechos Humanos. Iniciativa Latinoamericana para el avance de los Derechos Humanos de las Mujeres II. Mujeres, Ciudadanía y Multiculturalismo. Santiago de Chile: Centro de Derechos Humanos, Facultad de Derecho, Universidad de Chile, 2012.

GONZÁLEZ, Marianne y NASH, Claudio (Eds.). Transparencia, Lucha contra la Corrupción y el Sistema Interamericano de Derechos Humanos. Santiago de Chile: Centro de Derechos Humanos, Facultad de Derecho, Universidad de Chile, 2012.

SANDOVAL, Marcela (Comp.). Justicia, Género y Sexualidad. Santiago de Chile: Centro de Derechos Humanos, Facultad de Derecho, Universidad de Chile - Red Alas, 2012.

NASH, Claudio; GONZÁLEZ, Camila y NOGUEIRA, Andrés (Eds.). Informe - Diagnóstico sobre la incorporación de los derechos humanos en las carreras de Derecho en Chile. Santiago de Chile: Centro de Derechos Humanos, Facultad de Derecho, Universidad de Chile - Instituto Nacional de Derechos Humanos, 2011.

MEDINA, Cecilia y NASH, Claudio. Sistema interamericano de derechos humanos: Introducción a sus mecanismos de protección. Santiago de Chile: Centro de Derechos Humanos, Facultad de Derecho, Universidad de Chile, 2011.

BAZÁN, Víctor y NASH, Claudio (Eds.). Justicia constitucional y derechos fundamentales. Fuerza normativa de la Constitución. 2010. Montevideo: Konrad-Adenauer-Stiftung E.V. - Centro de Derechos Humanos, Facultad de Derecho, Universidad de Chile, 2011. 
GARRETÓN, Francisca; GONZÁLEZ, Marianne y LAUZÁN, Silvana. Estudio de políticas públicas de verdad y memoria en 7 países de América Latina. Santiago de Chile: Centro de Derechos Humanos, Facultad de Derecho, Universidad de Chile, 2011.

PALACIOS, Patricia. El tratamiento de la violencia de género por la Organización de Naciones Unidas. Santiago de Chile: Centro de Derechos Humanos, Facultad de Derecho, Universidad de Chile, 2011.

BAZÁN, Víctor y NASH, Claudio (Eds.). Justicia constitucional y derechos fundamentales. Aportes de Argentina, Bolivia, Brasil, Chile, Perú, Uruguay y Venezuela. 2009. Montevideo: KonradAdenauer-Stiftung E.V. - Centro de Derechos Humanos, Facultad de Derecho, Universidad de Chile, 2010.

NASH, Claudio y MUJICA Ignacio (Eds.). Derechos humanos y juicio justo. Lima: Colegio de las Américas (COLAM) - Organización Universitaria Interamericana (OUI IOHE), 2010.

$\mathrm{NASH}$, Claudio. Las Reparaciones ante la Corte Interamericana de Derechos Humanos (19882007). Santiago de Chile: Centro de Derechos Humanos, Facultad de Derecho, Universidad

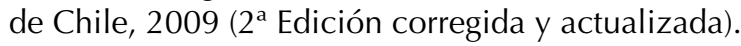

LAUZÁN, Silvana y TORO, Constanza. Anti-Corrupción. Actualizando los desafíos. Informe sobre la segunda reunión regional de especialistas en transparencia en América Latina. Santiago de Chile: Centro de Derechos Humanos, Facultad de Derecho, Universidad de Chile, 2009.

SANDOVAL, Marcela (Red.). Iniciativa Latinoamericana para el Avance de los Derechos Humanos de las Mujeres. Santiago de Chile: Centro de Derechos Humanos, Facultad de Derecho, Universidad de Chile, 2009.

TRABUCCO, Alia (Ed.). Derechos humanos hoy. Balance internacional. Santiago de Chile: Editorial Catalonia - Centro de Derechos Humanos, Facultad de Derecho, Universidad de Chile, 2008.

Centro de Derechos Humanos. Transparencia, accountability y lucha contra la corrupción - Syllabus $C D$. Santiago de Chile: Centro de Derechos Humanos, Facultad de Derecho, Universidad de Chile, 2008.

ZALAQUETT, José y MUÑOZ, Alex (Eds.). Transparencia y probidad pública: Estudios de caso de América Latina. Santiago de Chile: Centro de Derechos Humanos, Facultad de Derecho, Universidad de Chile, 2008.

MEDINA, Cecilia y NASH, Claudio. Sistema interamericano de derechos humanos: Introducción a sus mecanismos de protección. Santiago de Chile: Centro de Derechos Humanos, Facultad de Derecho, Universidad de Chile, 2007.

PALACIOS, Patricia. Selección de jurisprudencia universal e interamericana en materia de derechos humanos y mujeres. Santiago de Chile: Centro de Derechos Humanos, Facultad de Derecho, Universidad de Chile, 2006.

PALACIOS, Patricia. La no discriminación. Estudio de la jurisprudencia del Comité de Derechos Humanos sobre la cláusula autónoma de no discriminación. Santiago de Chile: Centro de Derechos Humanos, Facultad de Derecho, Universidad de Chile, 2006.

PALACIOS, Patricia. Las convenciones internacionales de derechos humanos y la perspectiva de género. Santiago de Chile: Centro de Derechos Humanos, Facultad de Derecho, Universidad de Chile, 2005. 
ZALAQUETT, José (Red.). Transparencia, rendición de cuentas y lucha contra la corrupción en América. Santiago de Chile: Centro de Derechos Humanos, Facultad de Derecho, Universidad de Chile, 2005.

MEDINA, Cecilia. La Convención Americana: Teoría y jurisprudencia. Vida, integridad personal, libertad personal, debido proceso y recurso judicial. San José de Costa Rica: Mundo Gráfico Centro de Derechos Humanos, Facultad de Derecho, Universidad de Chile, 2005.

Centro de Derechos Humanos. 18 Ensayos. Justicia transicional, Estado de Derecho y democracia. Santiago de Chile: Centro de Derechos Humanos, Facultad de Derecho, Universidad de Chile - Fundación Sueca para los Derechos Humanos, 2005.

Centro de Derechos Humanos. Compilación de Observaciones finales del Comité de Derechos Humanos sobre países de América Latina y el Caribe (1977-2004). Santiago de Chile: Oficina del Alto Comisionado de las Naciones Unidas para los Derechos Humanos - Centro de Derechos Humanos, Facultad de Derecho, Universidad de Chile, 2005.

NASH, Claudio. Las reparaciones ante la Corte Interamericana de Derechos Humanos. Santiago de Chile: Centro de Derechos Humanos, Facultad de Derecho, Universidad de Chile - Fundación Ford, 2004.

ZALAQUETT, José (Ed.). Temas de derechos humanos en debate. Santiago de Chile: Instituto de Defensa Legal (IDL) - Centro de Derechos Humanos, Facultad de Derecho, Universidad de Chile, 2004.

Defensoría Penal Pública. Manual de Derecho Internacional de los Derechos Humanos para Defensores Penales Públicos. Santiago de Chile: Centro de Documentación Defensoría Penal Pública, 2003. 
